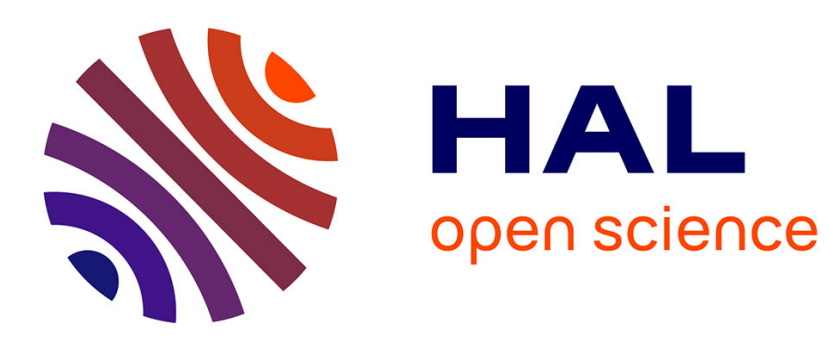

\title{
Energétique du formage magnétique
}

\author{
C. Rioux, F. Rioux-Damidau
}

\section{To cite this version:}

C. Rioux, F. Rioux-Damidau. Energétique du formage magnétique. Revue de Physique Appliquée, 1987, 22 (4), pp.253-259. 10.1051/rphysap:01987002204025300 . jpa-00245538

\section{HAL Id: jpa-00245538 https://hal.science/jpa-00245538}

Submitted on 1 Jan 1987

HAL is a multi-disciplinary open access archive for the deposit and dissemination of scientific research documents, whether they are published or not. The documents may come from teaching and research institutions in France or abroad, or from public or private research centers.
L'archive ouverte pluridisciplinaire HAL, est destinée au dépôt et à la diffusion de documents scientifiques de niveau recherche, publiés ou non, émanant des établissements d'enseignement et de recherche français ou étrangers, des laboratoires publics ou privés. 


\title{
Energétique du formage magnétique
}

\author{
C. Rioux et F. Rioux-Damidau \\ Laboratoire d'Electrotechnique des Universités Paris VI et XI, Unité associée au CNRS n 845, 91405 Orsay, \\ France
}

(Reçu le 30 septembre 1986, accepté le 18 décembre 1986)

\begin{abstract}
Résumé. - On détermine les ordres de grandeurs de la durée et de l'intensité des impulsions d'énergie à mettre en œuvre pour former des plaques métalliques de diverses épaisseurs.
\end{abstract}

\begin{abstract}
One determines the approximative values of the duration and intensity of energy pulses which are necessary for the magnetic forming of metallic plates of different thicknesses.
\end{abstract}

La technique du formage magnétique consiste, comme il est bien connu, à lancer à l'aide d'efforts électromagnétiques intenses une pièce métallique sur une matrice ; la vitesse doit être suffisante pour qu'elle en épouse la contre-forme mais pas trop élevée afin qu'elle ne s'y incruste pas. Dans ce dernier cas, d'ailleurs, on obtient un placage qui est une extension de la technique précédente. Dans la pratique habituelle, la pièce est placée près d'une bobine parcourue par un courant mono-impulsionnel ou alternatif, fourni par un banc de condensateurs ; elle est alors le siège de courants induits qui se créent d'autant plus facilement que le matériau est bon conducteur. Les courants induits sont soumis à d'importantes forces de Laplace qui propulsent la plaque. L'énergie du banc de condensateurs détermine l'énergie cinétique transmissible à la pièce et donc ses dimensions maximales. De fait, les bancs utilisés sont de l'ordre de $10 \mathrm{~kJ}$ [1-3] et atteignent exceptionnellement le MJ [4]: la technique du magnéto-formage est ainsi réservée aux pièces en matériau bon conducteur (cuivre ou aluminium) de dimensions modestes.

Aujourd'hui, les sources impulsionnelles d'énergie à base de stockage cinétique pourraient fournir des énergies très élevées à bas prix. Elles seraient typiquement constituées d'un volant, d'une génératrice impulsionnelle $[5,6]$, d'une bobine de stockage magnétique et d'un système de transfert inductif. Par ailleurs, les condensateurs voient aussi leurs performances s'accroître et deviennent de plus en plus accessibles au niveau ou au-delà du MJ. On peut donc envisager d'appliquer la technique du magnéto-formage à des matériaux moins bon

REVUE DE PHYSIQUE APPLIQUÉE. - T. 22, N`4, AVRIL 1987 conducteurs et même magnétiques tels que l'acier et à des pièces de dimensions quelconques.

Nous nous proposons ici de préciser les conditions énergétiques de ce procédé en particulier pour les hautes énergies. Nous examinerons donc :

- la création des courants induits

- la mise en vitesse des pièces

- la valeur de la vitesse à atteindre

et en déduirons, en ordre de grandeur, les caractéristiques de l'impulsion d'énergie nécessaire pour une pièce de nature et dimensions données.

\section{Création des courants induits}

Le système le plus classique est celui constitué par une plaque d'épaisseur $e$, de conductivité $\sigma$ et de perméabilité $\mu$ placée tout près d'une bobine ayant la forme d'une spirale (Fig. 1).

La détermination précise des courants induits dans la plaque est un problème difficile car très

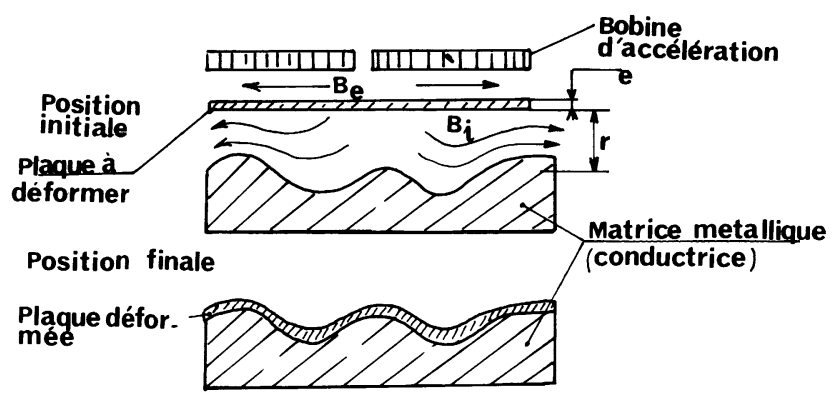

Fig. 1. - Formage magnétique d'une plaque.

[Magnetic forming of a plate.] 
dépendant des conditions aux limites. De manière générale, l'accélération de la plaque est efficace chaque fois que le champ créé entre la bobine d'accélération et la plaque est nettement supérieur (par exemple d'un facteur dépassant 2) à celui qui règne de l'autre côté de la plaque. A titre d'exemple, dans le cas d'école où l'objet à accélérer est constitué par un cylindre conducteur creux et illimité (de rayon $r$ et d'épaisseur $e \ll r$ ), la constante de temps de diffusion du champ au travers du cylindre (Fig. 2) est $\tau_{D}=\mu_{0} \sigma e r$ [7]. En régime de mono-impulsion,

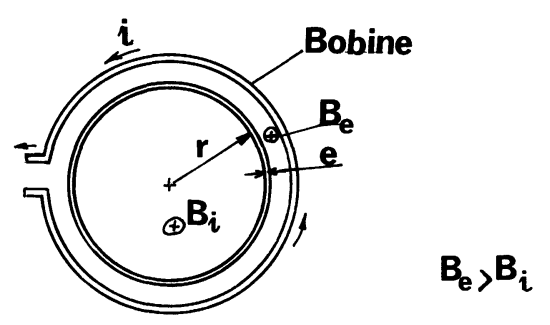

Fig. 2. - Formage magnétique d'un tube.

[Magnetic forming of a tube.]

la diffusion du champ reste acceptable si le temps de mise en vitesse est de l'ordre ou inférieur à $\tau_{\mathrm{D}}$. En régime sinusoïdal de pulsation $\omega$, il faut satisfaire la condition $\omega \tau_{\mathrm{D}} \geqslant 1$. Dans la pratique, le courant fourni par un banc de condensateurs est oscillatoire amorti mais les conditions restent sensiblement les mêmes [8]. Lorsque l'objet conducteur à accélérer a une forme quelconque (par exemple une plaque), on peut se ramener grosso modo à un schéma du même type : $e$ représente l'épaisseur moyenne de la plaque tandis que $r$ est assimilable à la distance séparant la plaque de la grosse masse conductrice la plus voisine (voir Fig. 1).

\section{Vitesse de formage.}

Dans la technique du magnéto-formage, la forme s'obtient lors de l'arrêt brusque sur une matrice de la pièce à laquelle on a communiqué une vitesse suffisante (Fig. 1) : la déformation du matériau est donc du type rapide et non quasi statique. Les contraintes qui apparaissent dans ces conditions peuvent alors être notablement supérieures à celles obtenues lors des déformations statiques [9-11], et commencent seulement à être connues [12] ; elles font intervenir un grand nombre de paramètres tels que les orientations cristallines, la présence d'inclusions, etc... La vitesse que doit posséder le matériau à l'arrivée sur la matrice est donc difficile à préciser. Cependant, pour évaluer un premier ordre de grandeur, nous allons considérer que le métal conserve ses caractéristiques quasi statiques même en dynamique (l'effort réel en cours de déformation pouvant parfois être doublé).
Considérons donc une plaque métallique, d'épaisseur $e$, de masse spécifique $\bar{\omega}$ et une matrice dans laquelle un trou de diamètre $l$ a été percé avec $l \gg e$. Quand la plaque arrive à la vitesse $v$ sur la matrice, son énergie cinétique se transforme en travail de déformation plastique et l'on a :

$$
\frac{1}{2} \bar{\omega} v^{2}=R A
$$

où $R$ est la contrainte à la rupture et $A$ l'allongement à la rupture obtenus lors d'essais de traction quasi statique.

On en déduit :

$$
\begin{aligned}
& v=140 \mathrm{~m} / \mathrm{s} \text { pour le cuivre } \\
& v=150 \mathrm{~m} / \mathrm{s} \text { pour l'aluminium } \\
& v=150 \mathrm{~m} / \mathrm{s} \text { pour un acier courant } .
\end{aligned}
$$

L'expérience a montré qu'à une vitesse de $140 \mathrm{~m} / \mathrm{s}$ une pièce d'aluminium de $1 \mathrm{~mm}$ d'épaisseur se déforme effectivement [13].

Des expériences effectuées avec de l'acier projeté sur une matrice comportant des trous ont montré qu'une vitesse de $100 \mathrm{~m} / \mathrm{s}$ permet d'obtenir des déformations importantes sur des épaisseurs faibles ou des déformations faibles sur des épaisseurs plus importantes. Une vitesse de $150 \mathrm{~m} / \mathrm{s}$ permet d'obtenir des déformations régulières et assez profondes sur plaques épaisses [14].

On peut aussi rechercher des taux d'allongements très importants, jusqu'à $80 \%$, et il est alors nécessaire de mettre en œuvre des vitesses très élevées, atteignant jusqu'à $500 \mathrm{~m} / \mathrm{s}$ [4]. Finalement, il est difficile de définir précisément une «vitesse nécessaire pour le formage » puisqu'elle dépend en particulier de l'amplitude de déformation cherchée, mais il semble que 100 à $200 \mathrm{~m} / \mathrm{s}$ soit un ordre de grandeur tout à fait réaliste.

\section{Energétique.}

Cette étude étant axée vers l'utilisation ultérieure de sources d'énergie à base de stockage cinétique et de disjoncteurs ou de transfert inductif à haut rendement $[15,16]$, nous nous sommes limités à la propulsion de plaques métalliques par mono-impulsions de courant, la bobine étant elle-même alimentée par une inductance fixe (l'alimentation possible par inductances variables n'est pas envisagée ici).

3.1 LE CIRCUIT. - Pour que la propulsion soit efficace, nous savons que le champ ne doit pas diffuser au travers de la plaque de manière trop importante : cette dernière constitue ainsi un quasiécran vis-à-vis du champ magnétique. Le courant total qui circule dans la plaque est alors voisin de celui qui circule dans la bobine: ils constituent ensemble deux nappes de courant de sens opposés situées en vis-à-vis. Tout se pásse au niveau des bornes de la bobine comme si le système était 
quasiment constitué par deux bobines connectées en série et mobiles l'une par rapport à l'autre. La prise en compte des conditions aux limites étant difficile (parce que spécifique à chaque problème traité) et assez peu déterminante en pratique (la diffusion est faible en général), nous avons finalement supposé que :

- la bobine est mono-spire (dans la pratique, il s'agissait d'effectuer les adaptations d'impédance nécessaires),

- la plaque est parcourue par un courant égal à celui de la bobine (absence de tout phénomène de diffusion dans la plaque ; les pertes, assez faibles, sont prises en compte par la résistance de la plaque).

Des coefficients de correction prennent en compte dans le calcul l'épaisseur de peau $\delta$ et la largeur finie de la plaque : ils seront discutés à la fin des résultats.

La bobine " mobile » (plaque) est caractérisée par ses dimensions géométriques (épaisseur $e$, largeur $d$, longueur $h$ ), sa densité $\bar{\omega}$ et sa conductivité $\sigma$. Le système est alimenté à partir d'une inductance de stockage transitoire de valeur $L_{\mathrm{f}}$, préalablement chargée en énergie à l'aide d'une source impulsionnelle d'énergie. Le schéma du circuit est alors représenté sur la figure 3 .

L'instant initial $t_{0}$ est évidemment choisi au moment où le courant induit par la source d'énergie dans l'inductance $L_{\mathrm{f}}$ atteint sa valeur maximale $I_{\mathrm{M}}$. L'énergie stockée est alors :

$$
W_{\mathrm{i}}=\frac{1}{2} L_{\mathrm{f}} I_{\mathrm{M}}^{2}
$$

On ouvre le disjoncteur $D$. Le courant est transféré vers l'ensemble bobine-plaque et la plaque commence à se déplacer. Soient $L_{\mathrm{p} 0}$ l'inductance de l'ensemble bobine-plaque à l'instant $t_{0}$ de la commutation (elle est faible puisque la plaque est initialement très proche de la bobine) et $L$ sa valeur à l'instant $t$. Si $x$ est le déplacement et $x_{0}$ la distance apparente initiale des bobines, on a :

$$
\begin{aligned}
\frac{L_{\mathrm{p}}}{L_{\mathrm{p} 0}} & =1+\frac{x}{x_{0}}, \\
L_{\mathrm{p} 0} & =\mu_{0} \frac{x_{0} h}{d} .
\end{aligned}
$$

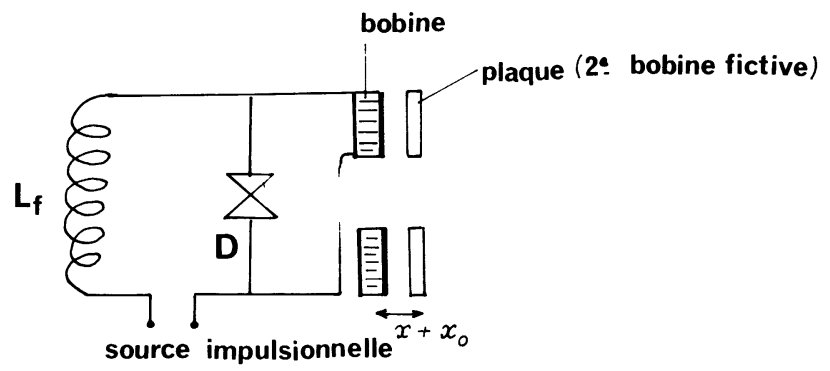

Fig. 3. - Circuit électrique.

[Electrical circuit.]
L'inductance totale du circuit au temps $t$ est :

$L(t)=L_{\mathrm{f}}+L_{\mathrm{p}}=L_{0}\left(1+\frac{L_{\mathrm{p} 0}}{L_{0}} \cdot \frac{x}{x_{0}}\right)=L_{0}(1+Y)$

avec

$$
L_{0}=L_{\mathrm{f}}+L_{\mathrm{p} 0}
$$

et

$$
Y=\frac{L_{\mathrm{p} 0}}{L_{0}} \cdot \frac{x}{x_{0}}=\frac{\mu_{0} h}{L_{0} d} x
$$

Durant la commutation qui est très brève, l'énergie perdue est :

$$
W_{\mathrm{d}} \# W_{\mathrm{i}} \cdot \frac{L_{\mathrm{p} 0}}{L_{0}}
$$

et l'énergie disponible à $t_{0}$ :

$$
W_{0}=W_{\mathrm{i}} \cdot \frac{L_{\mathrm{f}}}{L_{0}}=\frac{1}{2} L_{0} I_{0}^{2} .
$$

\subsection{EQUATIONS D'ÉVOLUTION.}

3.2.1 Bilan énergétique. - Nous tiendrons compte des pertes par effet Joule dans la résistance $R$ de la plaque mais négligerons les autres résistances. Le bilan énergétique à l'instant $t$ s'écrit :

$$
\frac{1}{2} L_{0} I_{0}^{2}=\frac{1}{2} L I^{2}+\frac{1}{2} \bar{\omega} e d h v^{2}+R_{0} \int_{t_{0}}^{t} I^{2}(t) \mathrm{d} t
$$

où $v$ est la vitesse de la plaque.

Nous supposons la résistance $R_{0}$ constante et admettons qu'elle est donnée par l'expression :

$$
R_{0}=\frac{1}{\sigma} \cdot \frac{h}{\delta d}
$$

$\delta$ étant l'épaisseur de peau.

Nous supposons aussi que les échauffements sont suffisamment faibles pour que la conductivité $\sigma$ reste constante.

La vitesse acquise sous l'action de la pression magnétique $p_{\mathrm{M}}$ est telle que

$$
\bar{\omega} \cdot e \frac{\mathrm{d} v}{\mathrm{~d} t}=p_{\mathrm{M}} \text {. }
$$

Le champ ne diffusant pas et la plaque étant supposée large, l'induction magnétique vaut $B=\mu_{0} I / d$ et la pression magnétique $p_{\mathrm{M}}=$ $\mu_{0} I^{2} / 2 d^{2}$. En pratique, cette valeur maximale n'est pas atteinte. Nous en tiendrons compte en écrivant :

$$
p_{\mathrm{M}}=\gamma_{0} I^{2} / 2 d^{2}
$$

avec $\gamma<1$ et nous aurons :

$$
v=\frac{\mu_{0} \gamma}{2 \bar{\omega} e d^{2}} \int_{t_{0}}^{t} I^{2}(t) \mathrm{d} t
$$


Lorsque la plaque est plus épaisse que l'épaisseur de peau en régime exponentiel, soit $e \gg \delta$ avec $\delta^{2} \# T / \mu_{0} \sigma$ ( $T$ étant le temps de propulsion de la plaque), le terme Joule ainsi est lié à la vitesse par la relation :

$$
R_{0} \int_{t_{0}}^{t} I^{2} \mathrm{~d} t=\frac{2}{\mu_{0} \sigma} \bar{\omega} h d \frac{e}{\delta \gamma} v .
$$

Ainsi, l'équation (11) s'écrit :

$$
L_{0} I_{0}^{2}-L I^{2}=\bar{\omega} e d h v^{2}+\frac{4}{\mu_{0} \sigma} \bar{\omega} h d \frac{e}{\delta \gamma} v .
$$

On posera alors :

$$
v_{\mathrm{D}}=\frac{1}{\mu_{0} \sigma \delta \gamma} .
$$

Ce terme, qui a la dimension d'une vitesse, caractérise la vitesse de diffusion du champ. Dans le tableau I, nous avons groupé les valeurs de $v_{\mathrm{D}}$ pour plusieurs métaux, en supposant $\delta=1 \mathrm{~mm}$ et $\gamma=1$.

Tableau I. - Vitesse de diffusion pour divers métaux.

[Diffusion velocity for different metals.]

$$
\begin{array}{l|r|r|r} 
& \mathrm{Cu} & \mathrm{Al} & \text { Acier } \\
\sigma: 10^{7} \Omega^{-1} \mathrm{~m}^{-1} & \overline{-} & \overline{-} & - \\
v_{\mathrm{D}}: \mathrm{m} / \mathrm{s} & 5,5 & 3,7 & 0,67 \\
& 14,3 & 21,5 & 119
\end{array}
$$

On posera aussi :

$$
v_{\mathrm{i}}^{2}=\frac{L_{0} I_{0}^{2}}{\bar{\omega} e d h}
$$

$v_{\mathrm{i}}$ représente la vitesse que pourrait prendre la plaque si toute l'énergie initiale était convertie en énergie cinétique.

L'équation (16) prend dans ces conditions la forme :

$$
v^{2}+4 v v_{\mathrm{D}}=v_{\mathrm{i}}^{2}\left(1-\frac{L I^{2}}{L_{0} I_{0}^{2}}\right)
$$

3.2.2 Evolution du courant. - Pour résoudre l'équation (19), il nous faut donc connaître le courant $I(t)$. On écrit à cet effet :

$$
\frac{\mathrm{d}}{\mathrm{d} t}(L I)+R_{0} I=0
$$

soit, compte tenu de (7) et de (8),

$$
\begin{gathered}
\left(L_{0} \frac{\mathrm{d} Y}{\mathrm{~d} t}+R_{0}\right) I+L_{0}(1+Y) \frac{\mathrm{d} Y}{\mathrm{~d} t}=0 \\
\frac{\mathrm{d} Y}{\mathrm{~d} t}=\frac{\mu_{0} h}{L_{0} d} v .
\end{gathered}
$$

3.2.3 Echauffement. - Il est intéressant aussi d'évaluer l'échauffement $\theta$ de la plaque. Nous garde- rons les hypothèses précédentes ( $\sigma$ constant, courant réparti sur l'épaisseur de peau $\delta$ ) en supposant implicitement l'échauffement faible. On a :

$$
\bar{\omega} c \theta=\int_{t_{0}}^{t} \frac{1}{\sigma}\left(\frac{I}{\delta d}\right)^{2} \mathrm{~d} t .
$$

En comparant avec (14) et (17) on en déduit

$$
c \theta=2 v v_{\mathrm{D}} \frac{e}{\delta} .
$$

3.2.4 Résolution. - L'ensemble des équations (19), (20), (21) et (22) a été transformé en posant:

$$
\begin{gathered}
V=\frac{v}{v_{\mathrm{i}}} \quad V_{\mathrm{D}}=\frac{v_{\mathrm{D}}}{v_{\mathrm{i}}} \\
\mathrm{d} T=\frac{\mathrm{d} t}{L_{0} / R_{0}} \quad j=\frac{I}{I_{0}} \quad, \\
\frac{\mathrm{d} j}{j}=-\frac{1+V / V_{\mathrm{D}}}{1+Y} \mathrm{~d} T \\
\mathrm{~d} Y=\frac{V}{V_{\mathrm{D}}} \mathrm{d} T \\
V^{2}+4 V V_{\mathrm{D}}=1-(1+Y) j^{2}
\end{gathered}
$$

avec comme conditions initiales

$$
j(0)=1, \quad V(0)=0, \quad Y(0)=0 .
$$

Le système (24) a été résolu numériquement par un procédé pas à pas.

3.3 RÉSUltats. - Les grandeurs qui nous intéressent sont celles à l'arrivée sur la matrice. En particulier, la vitesse $v$ doit alors être égale à la vitesse de formage.

La figure 4 donne la vitesse réduite $V$ en fonction

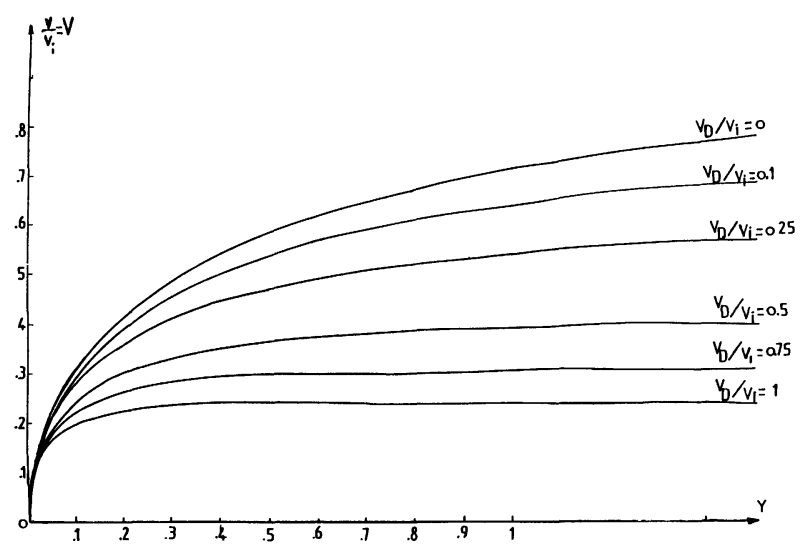

Fig. 4. - Vitesse réduite $v / v_{\mathrm{i}}$ en fonction du déplacement pour diverses valeurs de la vitesse de diffusion réduite $v_{\mathrm{D}} / v_{\mathrm{i}}$.

[Reduced velocity $v / v_{\mathrm{i}}$ as a function of the displacement for several values of the reduced diffusion velocity $v_{\mathrm{D}} / v_{\mathrm{i}}$.] 
du déplacement réduit $Y$ pour différentes valeurs de la vitesse de diffusion réduite $V_{\mathrm{D}}$. On constate que la vitesse atteinte par la plaque diminue lorsque la diffusion du champ augmente.

La figure 5 représente le temps réduit $T_{\mathrm{F}}$ en fonction de $v_{\mathrm{D}} / v$. Lorsque $v_{\mathrm{D}} / v \leq 1, T$ est proportionel à $v_{\mathrm{D}} / v$ :

$$
T_{\mathrm{F}}=1,6 Y v_{\mathrm{D}} / v
$$

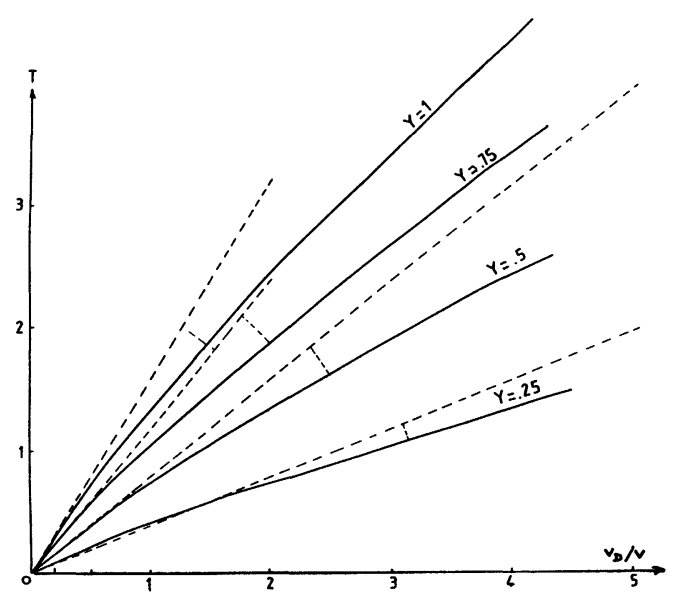

Fig. 5. - Temps de propulsion en unités réduites en fonction de $v_{\mathrm{D}} / v$.

[Reduced propulsion time as a function of $v_{\mathrm{D}} / v$.]

Cette condition est en général réalisée (cf. Tableau I) et le temps de propulsion de la plaque peut s'écrire :

$$
t \simeq 1,6 x / v \gamma
$$

La figure 6 donne $V^{2}=v^{2} / v_{\mathrm{i}}^{2}$ (c'est-à-dire le rendement $\eta$ ) en fonction de $v / v_{\mathrm{D}}$. On voit que $\eta$ croît avec $Y$ mais lentement à partir de $Y=0,75$. Comme $v / v_{\mathrm{D}}=\mu_{0} v \delta \gamma \sigma, \eta$ dépend en particulier de l'épaisseur de peau $\delta$ et du coefficient de forme $\gamma$.

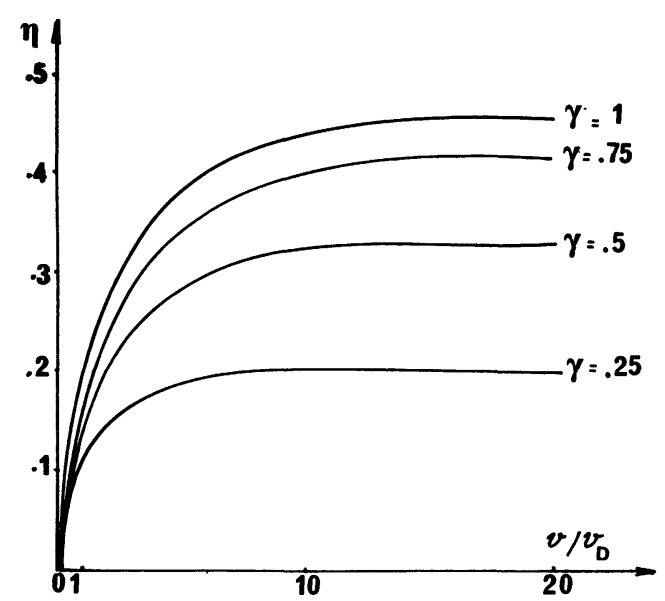

Fig. 6. - Rendement en fonction de $v / v_{\mathrm{D}}$.

[Efficiency as a function of $v / v_{\mathrm{D}}$.]

REVUE DE PHYSIQUE APPLIQUÉE. - T. 22, N 4, AVRIL 1987
3.4 DisCuSSION DES CORRECTIONS. - Deux éléments importants mais non essentiels n'ont pas été évalués numériquement :

- la diffusion du champ au travers de la plaque dans les systèmes inductifs (coefficient $\alpha$ ),

- les effets de largeur (coefficient $\gamma$ ).

En ce qui concerne la diffusion du champ, précisons que la pression magnétique sur la plaque est proportionnelle à $B_{\mathrm{i}}^{2}-B_{\mathrm{e}}^{2}$ (où $B_{\mathrm{i}}$ et $B_{\mathrm{e}}$ sont respectivement les valeurs de l'induction entre la bobine et la plaque et au-dehors de la plaque). Dans ces conditions, la propulsion reste efficace même avec des fuites de champ importantes. L'effet le plus important de la diffusion est de disperser les lignes de forces créées par la bobine, ce qui a pour effet de réduire $B_{\mathrm{i}}$ (la circulation de $H$ hors de la zone utile devient alors plus importante (voir Fig. 7)).

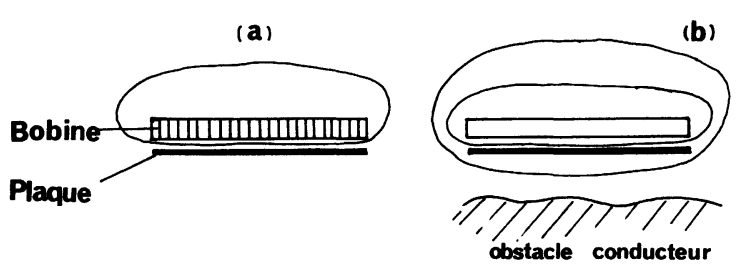

Fig. 7. - Allure des lignes de champ magnétique.

(a) : l'effet d'écran de la plaque est grand. Dans ce cas, toutes les lignes de force sont concentrées entre la bobine et la plaque. La circulation de $\mathbf{H}$ hors de cette région est faible ;

(b) : l'effet d'écran de la plaque est faible. Dans ce cas, le champ situé à l'extérieur de la plaque d'une part s'oppose à la pression du champ intérieur et d'autre part diminue sa valeur par l'accroissement de la circulation de $\mathbf{H}$ hors de la zone utile.

[Scheme of the magnetic field lines.]

Pour ce qui est de la largeur finie de la plaque, l'effet ne devient important que lorsque le chemin parcouru par cette dernière est de 10 à $20 \%$ de sa largeur. Au-delà, les efforts baissent beaucoup et nos calculs ne sont plus acceptables. Un traitement plus précis, mettant en œuvre l'environnement extérieur, sort du cadre de cette êtude générale.

\subsection{CARACTÉRISTIQUES DE LA SOURCE D'ÉNERGIE.}

- En choisissant $Y=0,75$ comme référence, on peut alors déduire de la figure 6 l'énergie à fournir par mètre carré de plaque :

$$
W=\frac{1}{2} \eta \bar{\omega} e v^{2}
$$

pour les divers métaux envisageables : cuivre, aluminium, acier. En pratique, on calcule plus précisément :

$$
\frac{\gamma}{\alpha} W=\frac{1}{2} \eta \bar{\omega} \delta \gamma v^{2}
$$


qui ne dépend que de $\delta \gamma \cdot \alpha=\frac{e}{\delta}$ est tel que $\alpha>1$ et le facteur de forme $\gamma$ tel que $\gamma<1$.

La figure 8 représente $\frac{\gamma}{\alpha} W$ en fonction de $\delta \gamma$ pour deux valeurs de $v$. Il apparaît nettement sur ces courbes que les sources de faible énergie ne permettent de former que le cuivre et l'aluminium de faible épaisseur (toujours dans le cadre des mono-impulsions étudiées ici).
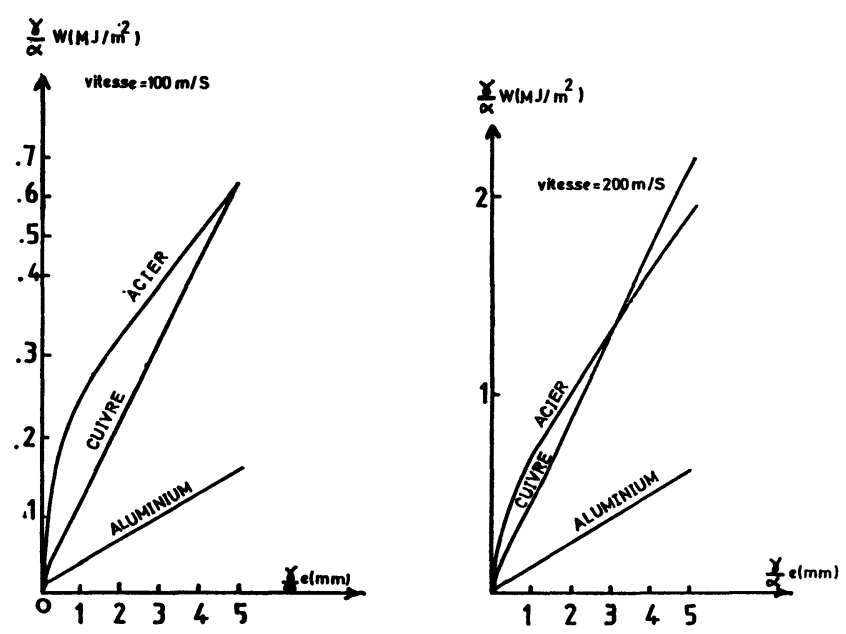

Fig. 8. - Energie de la source en fonction de l'épaisseur.

[Energy of the source as a function of the thickness.]

Pour que les efforts électromagnétiques soient importants, il faut que le champ diffuse peu et donc que $\alpha=e / \delta>1$. En fait, l'effet de peau est assez difficile à caractériser car nous avons supposé que le courant est fourni à la bobine au moment $t_{0}$ de la commutation sous forme d'un créneau et qu'ensuite l'intensité décroît pendant le temps $t_{\mathrm{F}}$ jusqu'à atteindre une valeur assez faible puisque la vitesse de la plaque est sensiblement stabilisée. Dans ces conditions, nous admettrons que l'ordre de grandeur de l'épaisseur de peau est celle que l'on aurait avec un courant décroissant exponentiellement avec la constante de temps $t_{\mathrm{F}}$ :

$$
\delta=\sqrt{\frac{t_{\mathrm{F}}}{\mu_{0} \sigma}}
$$

soit

$$
t_{\mathrm{F}}=\mu_{0} \sigma \delta^{2}=\frac{\mu_{0} \sigma e^{2}}{\alpha^{2}}
$$

Nous admettrons que la perméabilité de l'acier est voisine de $\mu_{0}$ car les inductions mises en jeu sont élevées. On peut ainsi calculer $t_{\mathrm{F}}$ (ou plutôt $\alpha^{2} t_{\mathrm{F}}$ ) c'est-à-dire le temps de délivrance de l'énergie par la source. Les valeurs obtenues sont regroupées dans le tableau II.
Tableau II. - Valeurs de $\alpha^{2} t_{\mathrm{F}}$ en $\mu \mathrm{s}$.

[Values of $\alpha^{2} t_{\mathrm{F}}$ in $\mu \mathrm{s}$.]

$$
\begin{array}{c|r|r|r}
e(\mathrm{~mm}) & \mathrm{Cu} & \mathrm{Al} & \multicolumn{1}{|c}{\text { Acier }} \\
\hline 1 & - & - & - \\
2 & 70 & 46 & 8 \\
5 & 280 & 186 & 30 \\
& 1750 & 1160 & 200
\end{array}
$$

Remarquons que si $\alpha$ est choisi grand, c'est-à-dire si l'épaisseur de peau relative est faible, l'énergie à fournir augmente comme $\alpha$ et la durée de l'impulsion diminue comme $\alpha^{2}$, la puissance augmente donc comme $\alpha^{3}$. En fait, dans le cas où $\alpha$ s'accroît, $\gamma$ se rapproche de 1 et l'effet est un peu moins marqué puisque l'énergie et la puissance croissent comme $\alpha / \gamma$ et $\alpha^{3} / \gamma$.

3.6 ECHAUFFEMENT. - Vérifions si l'échauffement reste acceptable. Le tableau III donne quelques valeurs de $\theta$ calculées à partir de (22) pour divers matériaux et diverses épaisseurs dans le cas où $\delta \sim e$ et $\gamma \sim 1$.

Tableau III. - Echauffement obtenu lors du formage de diverses plaques.

[Heating during the magnetic forming of different plates.]

\begin{tabular}{c|c|c|c}
$\begin{array}{c}\text { Epaisseur } \\
\mathrm{mm}\end{array}$ & Cuivre & Aluminium & Acier \\
- & - & - & - \\
0,1 & $70 \mathrm{~K}$ & $50 \mathrm{~K}$ & $680 \mathrm{~K}$ \\
1 & $7 \mathrm{~K}$ & $5 \mathrm{~K}$ & $68 \mathrm{~K}$ \\
5 & $1,4 \mathrm{~K}$ & $1,2 \mathrm{~K}$ & $14 \mathrm{~K}$
\end{tabular}

On voit que l'augmentation de la température n'est pas très importante, sauf dans le cas de l'acier et pour une épaisseur extrêmement faible de l'ordre de $0,1 \mathrm{~mm}$.

\section{Conclusion.}

Par des calculs généraux, nous avons déterminé l'ordre de grandeur des énergies à mettre en œuvre pour effectuer du formage magnétique à forte énergie. Pour des plaques grandes, lourdes et épaisses et à forte déformation (tôle d'acier de $1 \mathrm{~m}^{2}$, d'épaisseur égale à $5 \mathrm{~mm}$ ), les énergies nettes sont de l'ordre de 2 MJ. Compte tenu des divers rendements et effets annexes mal pris en compte (énergie de déformation des matériaux, effets de largeur finie, fuites de flux, résistance de l'inductance et des lignes de transport, etc...), l'énergie brute à décharger dans la bobine de stockage primaire devrait être plus élevée, par exemple de $5 \mathrm{MJ}$ dans l'exemple cité plus haut. 
Si l'on désire former des plaques en acier plus épaisses (10 $\mathrm{mm}$ par exemple), il faut augmenter les énergies (10 MJ ?).

Une autre application non abordée ici est le placage de feuilles fines, nécessitant une vitesse de l'ordre de $1500 \mathrm{~m} / \mathrm{s}$; dans ce dernier cas, les énergies envisagées seraient plus élevées encore (quelques dizaines de MJ pour le placage de grandes surfaces).

Dans tous les cas, il apparaît que l'emploi de sources d'énergie impulsionnelle autres que des condensateurs (par exemple du stockage cinétique associé à des transferts inductifs d'énergie à haut rendement) sont indispensables pour progresser industriellement dans ce domaine.

\section{Bibliographie}

[1] LiNOL, J. M., Journées SEE sur le magnétoformage, Grenoble, 27 et 28 novembre 1986.

[2] Erdosi, J., Li Monath, Gobl, N. id.

[3] Bourgoin, B., id.

[4] Gelé, C., Sci. Techn. 10 (1984) 44.

[5] Rioux, C., Sultanem, F., J. Appl. Phys. 50 (1979) 574.

[6] Bullion, T. M., Zowarka, R. C., Aanskoos, T. A., Weldon, W. F., RYlander, H. G., woodson, H. H., Proc. $3^{\text {rd }}$ IEEE International Pulsed Power Conference, Albuquerque N. M. (USA), 1981, p. 126.

[7] James, T. E., Phillpot, J., Proc. IEEe Conf., Pub. $\mathrm{n}^{\circ}$ 61, Electrical Methods of Machinery, Forming and Coating, U.K. (17-19 March 1970).

[8] Cheron, R., Leroy, M., Revue Phys. Appl. 16 (1981) 205.

[9] Hauser, F. E., Simmons, J. A., Dorn, J. E., Strain
Rate Effects in plastic wave propagation response of metals to high velocity deformation. (1960) p. 93.

[10] Hahn, R., Antoni, B., Lucidarme, J., Rioux, C., Rioux-Damidau, F., Revue Phys. Appl. 11 (1976) 409.

[11] Renaud, J. Y., Leroy, M., C. R. Heb. Séan. Acad. Sci. 28, 1975.

[12] Dormeval, R., Journées SEE sur le magnétoformage, Grenoble, 27 et 28 novembre 1986.

[13] Leroy, M., RenAud, J. Y., Formage et soudage par champs magnétiques, Brochure publiée par l'ENSM (Nantes).

[14] Alyassir, T., Thèse de Docteur-Ingénieur, Paris VI (1980).

[15] Baget, R., Rioux-Damidau, F., Rev. Sci. Instrum. 50 (1979) 4.

[16] Rioux, C., Revue Phys. Appl. 10 (1975) 75. 